\title{
Use of In Vivo Biotinylated GST Fusion Proteins to Select Recombinant Antibodies
}

\author{
Cedric Blanc, Madeleine Zufferey, and Pierre Cosson
}

Department of Cell Physiology and Metabolism, Centre Médical Universitaire, University of Geneva, Geneva, Switzerland

\begin{abstract}
Summary
Over the last 20 years, continuous advances in the field of molecular biology have led to the development of new strategies to discover and produce monoclonal antibodies, notably by phage display. Here we describe a simple procedure for antibody selection that considerably reduces the undesired selection of non-specific antibodies based on the use of biotinylated GST proteins fused to a target antigenic sequence. This procedure was tested on a collection of 7 different targets and resulted in the selection of a high percentage (71\%) of antibodies specific for each target. This simple and effective in vitro procedure has great potential to replace animal immunization for the development of specific antibodies.
\end{abstract}

Keywords: antibody, phage display, glutathione S-transferase (GST), biotinylation

\section{Introduction}

Biomedical research and drug development rely heavily on the use of specific monoclonal antibodies. The hybridoma technology developed in the 1970s allows the production of monoclonal antibodies following animal immunization and immortalization of antibody-producing cells (Kohler and Milstein, 1975). Over the past twenty years new approaches to discover and produce specific monoclonal antibodies in Escherichia coli have been developed. Recombinant antibody technology has the potential to fully replace the use of animals to identify and produce specific antibodies. Because it is entirely done in vitro, recombinant antibody selection can also generate antibodies that cannot be produced easily by animal immunization. For example, this technique allows the production of conformation-specific antibodies (Haque and Tonks, 2012; Nizak et al., 2003). This technology also allows the production of human antibodies, which can be used for therapeutic purposes (Ahmad et al., 2012). However, to date, recombinant antibodies are seldom used in academic research laboratories, and most antibodies destined for fundamental biomedical research are still produced by immunizing animals. The aim of this study was to develop a simple and robust protocol for the isolation of large numbers of specific recombinant antibodies.

Today, one of the classical methods to elicit antibodies is to express antigen-binding fragments of antibodies in bacterial cells and to display them at the surface of phages, a technique referred to as phage display. This strategy was first used to display short peptide fragments at the surface of phages (Smith, 1985). Subsequently, the VH and VL fragments of the anti-lysozyme antibody were displayed at the surface of phages and shown to bind their antigen in this configuration, demonstrating that phage display could in principle be used to select specific antibodies. Since then large antigen-binding repertoires have been generated using a variety of phage display formats (de Haard et al., 1999; Nissim et al., 1994).

Today, the most common phage display strategy to obtain specific antibodies is to isolate from an antibody library $\left(10^{9}\right.$ $10^{10}$ different clones) the few phages binding the antigen of interest immobilized on beads. After 3 to 4 successive rounds of selection, the selected phage population is expected to contain a high percentage of antibody fragments specifically binding the target antigen. A variety of non-specific antibodies also can be selected, notably antibodies binding to the beads themselves, and this can significantly complicate the selection of the desired antibodies (Vodnik et al., 2011). Here we describe a phage display selection procedure making use of in vivo biotinylated GST fusion proteins as target antigens. The proposed strategy strongly increased the selection of specific antibodies.

\section{Materials and methods}

Production of GST fusion proteins

The coding sequence of the C-terminal region of the Dictyostelium Rh50-like protein (accession number C22953) was fused with the coding sequence of GST in the pGEX-3X plasmid (GE Healthcare Life Sciences) using the BamHI and EcoRI restriction sites as previously described (Benghezal et al., 2001). E. coli Sure bacteria were transformed with the pGEX-3X-Rh50 construct. In bacteria growing exponentially $\left(\mathrm{OD}_{600}, 0.5\right)$ at $37^{\circ} \mathrm{C}$

Received July 8, 2013; accepted in revised form September 26, 2013; Epub October 11, 2013; http://dx.doi.org/10.14573/altex.1307081 
in LB medium ( $10 \mathrm{~g} / \mathrm{l}$ bactopeptone, $5 \mathrm{~g} / \mathrm{l}$ yeast extract, $10 \mathrm{~g} / \mathrm{l} \mathrm{Na}$ $\mathrm{Cl}$ ) containing $100 \mu \mathrm{M}$ ampicillin, recombinant protein expression was induced by addition of $1 \mathrm{mM}$ IPTG. After $3 \mathrm{~h}$, bacteria were pelleted and resuspended in lysis buffer $(\mathrm{PBS}+1 \%$ Triton + aprotinin $10 \mu \mathrm{g} / \mathrm{ml}+$ leupeptin $20 \mu \mathrm{g} / \mathrm{ml}+$ iodoacetamide $1.8 \mathrm{mg} /$ $\mathrm{ml}+\mathrm{PMSF} 18 \mu \mathrm{g} / \mathrm{ml}$ ) and lysed by sonication. The GST-Rh50 protein was purified on glutathione-coupled sepharose beads (GE Healthcare Life Sciences), then eluted in $500 \mu 1$ PBS containing $10 \mathrm{mM}$ glutathione, dialyzed in PBS, and biotinylated in PBS containing $1 \mathrm{mg} / \mathrm{ml}$ NHS-Biotin (Socochim) for $30 \mathrm{~min}$ at RT. The biotinylation reaction was stopped by the addition of $40 \mathrm{mM} \mathrm{NH} 4 \mathrm{Cl}$. The biotinylated GST-Rh50 protein was adsorbed onto magnetic streptavidin beads (112.05D, Invitrogen) or magnetic glutathione-coated beads (V8611, Promega) for $1 \mathrm{~h}$ at $4^{\circ} \mathrm{C}$, then the beads were washed twice with $5 \mathrm{ml}$ PBS + Triton $1 \%$ and twice with $1 \mathrm{ml}$ PBS. The purified GST-Rh50 protein was stored at $4^{\circ} \mathrm{C}$ in $1 \mathrm{ml}$ PBS.

We constructed a vector, named pAN4-GST, for in vivo production of GST proteins fused to an N-terminal biotinylation tag (GLNDIFEAQKIEWHE) (Beckett et al., 1999) by amplifying the GST coding sequence from the pGEX-3X plasmid using the following oligonucleotides: GGGAAGGAGCTCGAGTGGTAGTGGTTCCCCTATACTAGGTTATTGGAAAAT and GGGAAGAAGCTTAGTCACGATGAATTCCCGGGGATC. The PCR product was digested with SacI and HindIII and subcloned in the corresponding sites in the pAN4 vector (Beckett et al., 1999). To construct the pAN4-GST-Phg1a vector, the sequence encoding a fragment of the Dictyostelium Phg1a protein (Cornillon et al., 2000) (accession number AJ318760; residues 38 to 128) was fused to the GST coding sequence using the BamHI and EcoRI restriction sites.

To produce biotinylated GST fusion protein, we introduced the pAN4-GST plasmids into AVB101 bacteria (cat n AVB101, Avidity company). This strain contains the pBirCam vector, an IPTG-inducible plasmid encoding the BirA biotin-ligase. Bacteria were grown at $37^{\circ} \mathrm{C}$ in TYH medium, $\mathrm{pH} 7.4(20 \mathrm{~g} / 1$ tryptone, $10 \mathrm{~g} / 1$ yeast extract, $11 \mathrm{~g} / \mathrm{l} \mathrm{HEPES}, 5 \mathrm{~g} / 1 \mathrm{NaCl}, 1 \mathrm{~g} / \mathrm{l}$ $\mathrm{MgSO}_{4}$ ) containing $100 \mu \mathrm{M}$ ampicillin. The production and the biotinylation of the GST fusion protein were induced by adding $1.5 \mathrm{mM}$ IPTG and $50 \mu \mathrm{M}$ biotin to the medium. After $3 \mathrm{~h}$ at $37^{\circ} \mathrm{C}$, bacteria were harvested by centrifugation, resuspended in lysis buffer and lysed by sonication. The biotinylated GST fusion protein was purified using glutathione magnetic beads or streptavidin magnetic beads.

\section{Selection of antibodies by phage display}

A semi-synthetic naive library of human origin, the Tomlinson I, J library, was kindly provided by MRC, Cambridge, UK. This library contains approximately $10^{9}$ independent $\mathrm{scF}$ V recombinant antibodies inserted in the pIT2 vector (de Wildt et al., 2000).

In order to isolate antibodies specific for a target peptide, the corresponding GST fusion protein was immobilized on glutathione-coated or streptavidin-coated magnetic beads. Three successive selection and amplification cycles were performed. In each selection cycle, the phage library was incubated first with naked beads in $1 \mathrm{ml}$ PBS $+0.1 \%$ Tween $20+2 \%$ milk for $1 \mathrm{~h}$ in a silicon-coated Eppendorf tube (cat no. 022431081, Eppen- dorf) on a rotating wheel at RT (preclearing A). The supernatant was collected and incubated with GST coated beads (preclearing B). The second supernatant was incubated for $1 \mathrm{~h}$ with beads coated with the GST-target fusion protein (selection step). The beads were resuspended in PBS-Tween (PBS $+0.1 \%$ Tween 20), transferred into a new Eppendorf tube and washed 15 times with $1 \mathrm{ml}$ PBS-Tween. After the last wash, bound phages were eluted in $1 \mathrm{ml} 100 \mathrm{mM}$ TEA (triethylamine, Fluka) for $10 \mathrm{~min}$ at RT. $500 \mu 1$ TEA was recovered and neutralized in $500 \mu 1$ Tris (1 M, pH 7.4). After a further $10 \mathrm{~min}$ incubation, the remaining $500 \mu 1$ TEA was recovered and transferred to the same neutralization tube. The rescue and amplification of selected phages was achieved by infecting TG1 bacteria. $10 \mathrm{ml}$ exponentially growing TG1 (OD $600,0.5)$ were infected with $750 \mu 1$ neutralized phages for $10 \mathrm{~min}$ at $37^{\circ} \mathrm{C}$. After infection, bacteria were pelleted for $10 \mathrm{~min}$ at $3,300 \mathrm{x} \mathrm{g}$ and resuspended in $1.8 \mathrm{ml}$ of 2xTY medium (16 g/l Tryptone, $10 \mathrm{~g} / \mathrm{l}$ yeast extract, $5 \mathrm{~g} / \mathrm{l} \mathrm{NaCl}$ ). Infected bacteria were plated on three $15 \mathrm{~cm} \mathrm{2xTY/Amp/Glc}$ Agar plates $(2 \mathrm{xTY}+1 \%$ glucose $+100 \mu \mathrm{g} / \mathrm{ml}$ ampicillin) and grown overnight at $30^{\circ} \mathrm{C}$. To determine the number of phages recovered from the beads, $10^{-1}, 10^{-2}$, and $10^{-3}$ dilutions of the infected bacteria were also deposited on 2xTY/Amp/Glc plates.

Bacteria carrying the selected phagemids were scraped from the plates in $6 \mathrm{ml} 2 \times \mathrm{xTY}$ containing $30 \%$ glycerol, and an aliquot was frozen at $-80^{\circ} \mathrm{C}$. To produce phages, bacteria were diluted to an $\mathrm{OD}_{600}$ of $0.05 \mathrm{in} 100 \mathrm{ml} 2 \mathrm{xTY} / \mathrm{Amp} / \mathrm{Glc}$ and incubated at $37^{\circ} \mathrm{C}$ until they reached the exponential growth phase $\left(\mathrm{OD}_{600}, 0.5\right)$. Helper phages $\left(5 \times 10^{10}\right.$ for $10 \mathrm{ml}$ bacterial culture) were added, gently mixed, and incubated without agitation for $30 \mathrm{~min}$ at $37^{\circ} \mathrm{C}$. Bacteria were then centrifuged, resuspended in $50 \mathrm{ml} 2 \mathrm{xTY} / \mathrm{Amp} / \mathrm{Kana}$ medium $(2 \mathrm{xTY}+100 \mu \mathrm{g} / \mathrm{ml}$ ampicillin $+50 \mu \mathrm{g} / \mathrm{ml}$ kanamycin, no glucose), and incubated overnight at $30^{\circ} \mathrm{C}$ with agitation $(250 \mathrm{rpm})$. The bacterial culture was centrifuged for $10 \mathrm{~min}$ at $10,800 \mathrm{~g}$ to remove bacteria. After addition of $10 \mathrm{ml}$ ice cold $\mathrm{PEG} / \mathrm{NaCl}$ solution $(30 \% \mathrm{PEG} 8000+2.5 \mathrm{M} \mathrm{NaCl})$ to the supernatant, phages were allowed to precipitate for $1 \mathrm{~h}$ at $4^{\circ} \mathrm{C}$. Precipitated phages were pelleted for $10 \mathrm{~min}$ at $10,800 \mathrm{x} \mathrm{g}$ and resuspended in $1 \mathrm{ml}$ PBS. The remaining debris was removed by centrifugation for $10 \mathrm{~min}$ at $11,600 \mathrm{x}$ g before the cleared phage suspension was used in the next round of selection.

Individual clones were picked from the last selection and grown overnight at $37^{\circ} \mathrm{C}$ in $2 \mathrm{ml} 2 \mathrm{xTY} / \mathrm{Amp} / \mathrm{Glc}$. Phagemids were extracted and sequenced.

\section{Recombinant antibody production in HeLa cells}

Selected scFvs were inserted into the pFUSE-mIgG-Fc2 plasmid (accession number FJ716124) (Moutel et al., 2009), allowing expression of a fusion protein composed of the $\mathrm{scFv}$ fused to a mouse $\mathrm{Fc}$ immunoglobulin region ( $\mathrm{scFv}-\mathrm{Fc}$ antibodies). The subcloning was performed using $\mathrm{NcoI}$ and NotI restriction sites.

HeLa cells were maintained in Dulbecco's Modified Eagle Medium (DMEM) containing 5\% heat-inactivated fetal calf serum (FCS) and 2 mM L-Glutamine. They were transfected with $20 \mu \mathrm{g}$ plasmid DNA after calcium phosphate precipitation (Graham and van der Eb, 1973). Medium was replaced with fresh medium $24 \mathrm{~h}$ after the transfection. The medium containing secreted antibodies was collected $72 \mathrm{~h}$ later and stored at $-20^{\circ} \mathrm{C}$. 


\section{ELISA assays}

Bacterial lysates containing GST or antigenic GST fusion proteins were incubated in a 96-well glutathione-coated plate (cat no. 15240, Thermo Scientific) for $1 \mathrm{~h}$. Each well was incubated for $1 \mathrm{~h}$ at RT with $\mathrm{PBS}+1 \% \mathrm{BSA}$. After three washes with $100 \mu 1 \mathrm{PBS}+0.5 \% \mathrm{BSA}+0.05 \%$ Tween, the primary antibody was added (50 $\mu \mathrm{l}$ per well) and incubated for $1 \mathrm{~h}$. After three washes, an HRP coupled anti-mouse IgG antibody was added (50 $\mu \mathrm{l}$ per well) and incubated for $1 \mathrm{~h}$. After three washes the HRP substrate (cat n ${ }^{\circ}$ DY999, BD System) was added (50 $\mu 1$ per well). The reaction was stopped after $5 \mathrm{~min}$ at RT by addition of $25 \mu 1 \mathrm{H}_{2} \mathrm{SO}_{4}(2 \mathrm{M})$. The intensity of the signal was measured at a wavelength of $450 \mathrm{~nm}$.

\section{Western blot}

Bacteria expressing GST or antigenic GST fusion proteins were lysed and proteins separated on a $13 \%$ polyacrylamide gel, then transferred to a nitrocellulose membrane (Invitrogen, Carlsbad, CA). When indicated, a lysate from Dictyostelium cells $\left(5 \times 10^{5}\right.$ cells per lane) was migrated on a $10 \%$ polyacrylamide gel. The membrane was blocked overnight at $4^{\circ} \mathrm{C}$ in PBS $+0.1 \%$ Tween

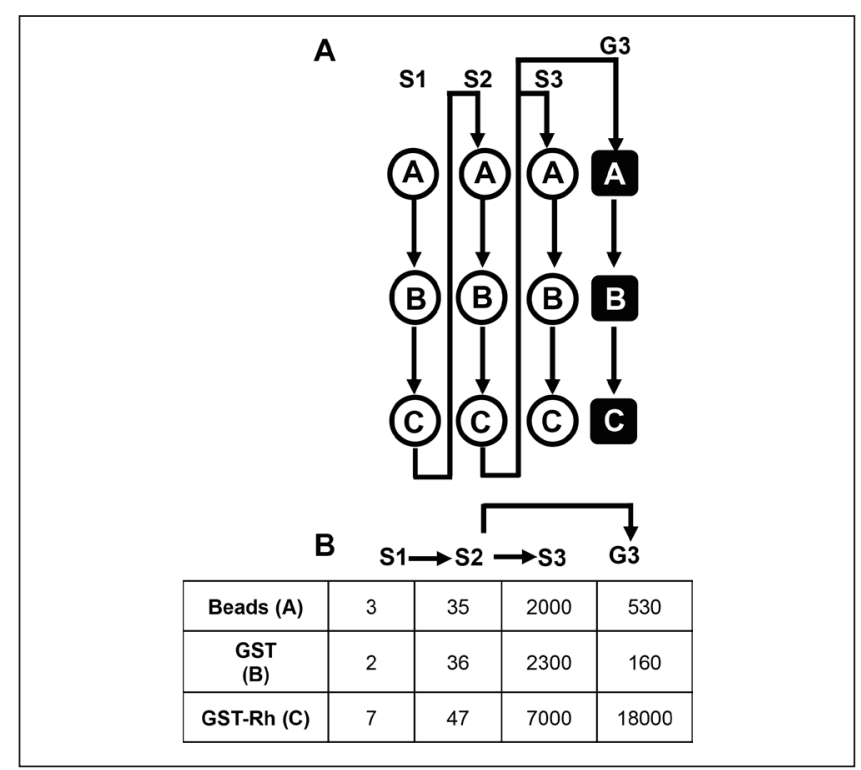

Fig. 1: A selection procedure eliminating non-specific scFv fragments

A: Two distinct procedures were tested to isolate scFv fragments specific for a target Rhesus sequence. The first strategy consisted in three successive selection rounds using biotinylated GST-Rh50 attached to streptavidin beads. In the second strategy, the first two selection rounds were done using GST-Rh50 attached to streptavidin beads, but the third selection round was done using GST-Rh50 attached to glutathione beads. B: For each selection round, the number of phages binding to beads (A), to GST-coated beads (B), and to GST-Rh50 coated beads (C) was evaluated and is indicated $\left(\times 10^{-4}\right)$. While repeated use of streptavidin beads led to the selection of phages binding streptavidin beads rather than GST-Rh50, performing the third selection round on glutathione beads allowed the specific selection of a vast majority of phages specifically binding the target Rhesus sequence.
$+7 \%$ milk. Then the membrane was washed for 10 min in PBSTween before incubation with the indicated recombinant antibody. After three washes in PBS-Tween, the membrane was incubated with an HRP-coupled anti-mouse Ig antibody (BioRad: cat $\left.n^{\circ} 170-6515\right)$, washed three times, and revealed by ECL (Amersham Biosciences).

\section{Results and discussion}

\section{Biotinylated GST fusion proteins for selection of recombinant antibodies}

In order to isolate antibodies recognizing the C-terminus of the Dictyostelium Rh50 protein (Benghezal et al., 2001), we expressed a GST-Rh50 protein in bacteria, purified and biotinylated it, and immobilized it either on glutathione-coated or on streptavidin-coated magnetic beads. As schematized in Figure 1A, phages were initially selected by three successive rounds of adsorption to GST-Rh50 bound to streptavidin-coated beads. At each selection step, phages were incubated first with glutathione-coated magnetic beads to eliminate phages binding to beads (preclearing A), then with GST-coated beads to eliminate antibodies binding to GST (preclearing B), and finally with antigen-coated beads to select Rh50-specific antibodies. The number of phages binding to beads, to GST-coated beads, and to GST-Rh50-coated beads was quantified.

While no significant variation in the number of phages binding to beads was seen in the first two cycles of enrichment, during the third cycle, we observed a clear increase in the number of phages binding to GST-Rh50-coated beads (Fig. 1B). However, this increase was paralleled by a similar increase in the number of phages binding streptavidin-coated beads or GSTcoated beads, indicating that this selection procedure largely selected phages binding to beads rather than to the target Rh50 sequence exposed on the beads. Selection of antibodies binding to avidin or binding non-specifically to beads was often reported in similar phage display screens (Vodnik et al., 2011).

In order to alleviate this problem, we tested an alternative selection procedure where phages were first allowed to bind GSTRh50 immobilized on streptavidin-coated beads for two rounds, and then, for the third round, to GST-Rh50 immobilized on glutathione-coated beads. We reasoned that antibodies binding streptavidin beads would be eliminated in this third round, leaving only antibodies binding specifically the GST-Rh50 protein. Indeed, phages selected on glutathione beads did not significantly bind beads, but a significant fraction of them specifically bound GST-Rh50 beads (Fig. 1B). The number of phages specifically binding GST-Rh50 was more than 30 times higher than that of phages binding naked beads or GST-coated beads. This result suggests that this procedure achieved a specific selection of phages recognizing the Rh50 antigen.

\section{In vivo biotinylation of GST fusion proteins}

The production and purification of GST fusion proteins can be arduous when production yields are low, or when the protein tends to aggregate. In addition, the efficiency of in vitro biotinylation can vary significantly, and most reagents used for in 


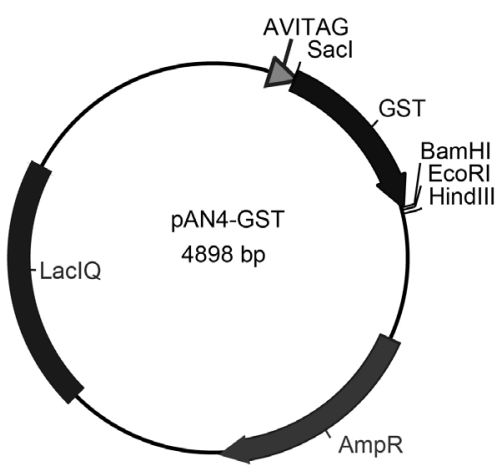

BamHI EcoRI Hindlll ggt cgt ggg atc ccc ggg aat tca tcg tga cta agc ttg $\begin{array}{llllllllll}G & R & G & \text { I } & P & G & N & S & S\end{array}$

Fig. 2: A plasmid allowing production of in vivo biotinylated GST fusion proteins

In the pAN4-GST vector, the coding sequence of the GST is preceded with an Avitag biotinylation sequence and followed by a cloning site where target antigenic sequences can be introduced. The sequence of the cloning site for insertion of target antigenic sequences is detailed.

vitro biotinylation (e.g., NHS-biotin) cannot be stably stored for prolonged periods of time. Finally, the random addition of a biotin moiety to a protein may destroy some of its antigenic sites. In order to avoid these potential problems, we developed a system to biotinylate GST fusion proteins in vivo on a defined site. For this, we made use of the in vivo biotinylation strategy of the Avidity company. This technique targets enzymatic conjugation of a single biotin on a unique 15 amino acid peptide tag (named Avitag) using the biotin ligase (BirA) from E. coli. We fused the Avitag-coding sequence at the 5' end of the GST coding sequence in the pAN4-GST plasmid (Fig. 2), allowing the production of biotinylated GST proteins in bacteria.

\begin{tabular}{|c|c|c|c|c|}
\hline A & \multicolumn{3}{|c|}{$s 1 \rightarrow s 2 \rightarrow s 3$} & G3 \\
\hline Beads & 8 & 64 & 3300 & 66 \\
\hline GST & 9 & 3 & 790 & 23 \\
\hline $\begin{array}{c}\text { GST- } \\
\text { Phg1a }\end{array}$ & 7 & 1 & 3400 & 2200 \\
\hline
\end{tabular}

B

\begin{tabular}{|c|c|c|c|}
\hline Beads & 15 & 110 & 30000 \\
\hline GST & 13 & 73 & 12000 \\
\hline $\begin{array}{c}\text { GST- } \\
\text { Phg1a }\end{array}$ & 9 & 50 & 10000 \\
\hline
\end{tabular}

Fig. 3: Selection of phages using in vivo biotinylated GST-Phg1A

As described in the legend of Figure 1, several strategies were tested for the isolation of specific scFv fragments using in vivo biotinylated GST-Phg1A as an antigen. A: Three successive rounds of selection using GST-Phg1A attached to streptavidin beads yielded a majority of clones binding to streptavidin beads. On the contrary, two selection rounds using streptavidin beads followed by one round using glutathione beads led to the isolation of a majority of phages specifically binding the target Phg1A sequence. B: Three successive rounds of selection using GST-Phg1A attached to glutathione beads led to the isolation of a majority of clones binding glutathione beads.

We then used in vivo biotinylated GST to isolate antibodies specific for a new epitope composed of 91 residues of the Dictyostelium Phg1A protein (Cornillon et al., 2000). The GST-Phg1A protein was produced and biotinylated in vivo as described above, and specific antibodies selected by phage display as described above for GST-Rh50. As observed for GST-Rh50, three successive rounds of selection using streptavidin-coated beads yielded phages binding non-specifically to streptavidin beads (Fig. 3A).

Tab. 1: Antigenic target sequences used in this study

\begin{tabular}{|c|c|c|}
\hline & Accession number & Antigenic sequence \\
\hline 1 : Dd Phg1a & AJ318760 & $\begin{array}{l}\text { GEEGAIKVNKITSVHTQIPYKYYQLPGVCQPKEGIIDDTENLGEILLGDRIENSDY } \\
\text { TFNFLTDGGKCKVINSESCSPIIKKEDLKVLEDRI }\end{array}$ \\
\hline 2 : Dd Mucolipin & EAL61621 & $\begin{array}{l}\text { VFISVYCISNIYIAIMESSYSRSIGIKSKLEKKAKEKKKLKEQYGDEINLDDHLWKT } \\
\text { LLGLKEEKLDDDDPNSNFNDDDDDDDDDDQSQSNLLNDDDYDDNDDDLDD } \\
\text { NNNNPNNNNSNNNNNNNNNNNNNKNDDDTSSNIS }\end{array}$ \\
\hline 3 : Dd SibA & Q54KF7 & KKSAPPTDAFFGEGAFADGAVSTNPMYEESGRSAINPLYEASSENL \\
\hline 4 : Dd mAM4 & Q54JQ7 & $\begin{array}{l}\text { SNNGKLSIFEKQSKRVIGDTFSLDLYYIIYKSIHTLNLKLLEIIILKNQSQYLNI } \\
\text { HSKLFTDQNDNYYLKNVDKIINNELIRFDKKTIELMKPLLKILKNKIN }\end{array}$ \\
\hline 5 : Dd NoxC & EAL61883 & CRYYTCLKTGGTKFYFHKENF \\
\hline $6:$ Dd TspB & Q55CW7 & ENFLKCCYWNSTSSRNPLLCPKDSKGIPKYTDTCDSVISSKISSN \\
\hline 7 : At AP4 & AAG60138 & LVRQMGVNPTSQNPTLFKDLLG \\
\hline
\end{tabular}




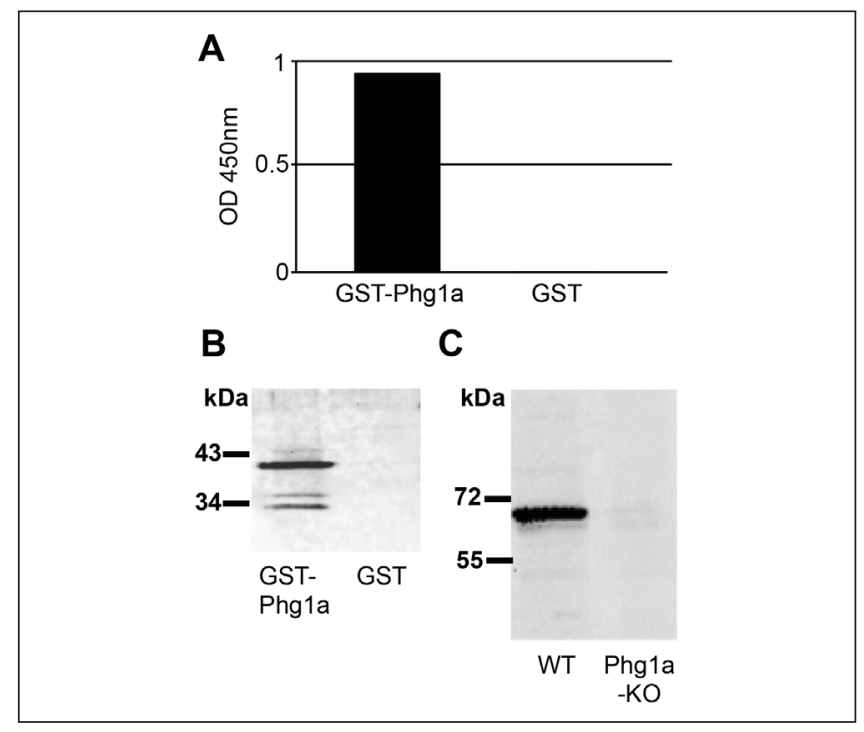

Fig. 4: A specific scFv fragment binding Phg1A

A: The P7 scFv fragment selected as described in the legend of Figure $3 \mathrm{~A}$ was subcloned into the pFUSE vector, the corresponding scFv-Fc fusion protein was produced in HeLa cells and tested by ELISA for its ability to specifically bind GST-Phg1A. The P7 antibody bound GST-Phg1A efficiently, but did not bind GST. B: Whole cell lysates of bacteria expressing either the GST or the GSTPhg1a proteins were separated in an SDS-PAGE, transferred to nitrocellulose, and incubated with the P7 antibody. The P7 antibody specifically bound GST-Phg1A, and not GST. C: A Dictyostelium cell lysate was separated in an SDS-PAGE and transferred to nitrocellulose. The P7 antibody bound a 70kDa protein, the size of the endogenous Phg1A protein, in a wild-type (WT) Dictyostelium cell lysate. Genetic disruption of the PHG1A gene (KO-Phg1a) led to the disappearance of the signal, confirming that the P7 antibody specifically recognized the endogenous Phg1a protein.

Conversely, three successive rounds of selection using glutathione-coated beads yielded phages binding non-specifically to glutathione beads (Fig. 3B). When only the third selection step was carried out using GST-Phg1A attached to glutathione-coated beads, selected phages bound efficiently to GST-Phg1A but not to glutathione-coated beads or to beads coated with GST (Fig. 3A).

In principle, the quantitative analysis described above indicates that the majority of the phages selected during the third round of selection should specifically bind the target Phgla sequence. To test further the specificity of the selected antibodies, eight individual clones were randomly chosen and sequenced. Seven of the eight clones were identical. We proceeded to test the ability of the corresponding antibody (named P7) to bind the
GST-Phg1a protein. For this, the scFv fragment was subcloned into the pFUSE-mFc2-adapt-scFv mammalian expression plasmid (Moutel et al., 2009), using NcoI and NotI restriction sites, allowing the expression of a scFv-Fc fusion in HeLa cells. The specific binding of this antibody to the GST-Phgla protein was tested by Western blot and ELISA. The P7 antibody bound specifically GST-Phg1A, but not GST, in an ELISA assay (Fig. 4A). In Western blot experiments, the P7 antibody specifically bound GST-Phg1A, and not GST, in a crude lysate of producing bacteria (Fig. 4B). In addition, when tested on a lysate of Dictyostelium cells, the P7 antibody recognized a band at approximately 70 $\mathrm{kDa}$, the size of the endogenous PhglA protein (Fig. 4C). This band was absent in a lysate of phg $1 \mathrm{~A} \mathrm{KO}$ cells (Fig. 4C), confirming that the P7 antibody specifically recognized the Dictyostelium Phg1A protein.

To assess the robustness of the selection scheme devised here, we used it on a series of target antigens. Table 1 describes the seven different target antigenic sequences used, six from Dictyostelium discoideum proteins (Mcln, Phg 1a, SibA, TspB, Mam4 and NoxC) and one from Arabidopsis protein (AP4). For each target we determined the number of undesirable phages as the sum of phages binding beads and phages binding beads coated with GST (U). We also measured the number of phages binding beads coated with the target GST fusion protein (T) (see Fig. 1). The specificity ratio $(\mathrm{T} / \mathrm{U})$ for each target is indicated in Table 2. The average specificity ratio was 10.2 (Tab. 2), indicating that this procedure efficiently selected antibodies specifically binding the target sequence. For each target, 1 to 5 selected $\mathrm{scFv}$ fragments were then subcloned, fused to an Fc sequence and produced in HeLa cells (Tab. 3). The resulting antibodies were tested by ELISA for their ability to bind their target. Over a total of $24 \mathrm{scFv}$ fragments tested, 17 (71\%) specifically bound the corresponding target antigen (Tab. 3). For all but one target (SibA), we obtained at least one specific antibody.

\section{Conclusion}

In this study, we describe a phage display selection procedure making use of an in vivo biotinylated GST fusion protein immobilized alternatively on streptavidin- and on glutathione-coated beads. We demonstrate that alternating selection of phages using antigen purified on streptavidin and on glutathione beads greatly reduces the selection of unspecific antibodies. Under the conditions used here, a large majority of non-specific antibodies binding to beads were selected when the same type of beads was used for all rounds of selection, while a majority of target-specific antibodies were selected when two types of beads were alter-

Tab. 2: Selection of specific antibodies to different antigenic targets

\begin{tabular}{|l|c|c|c|c|c|c|c|c|}
\hline Target No. & $\mathbf{1}$ & $\mathbf{2}$ & $\mathbf{3}$ & $\mathbf{4}$ & $\mathbf{5}$ & $\mathbf{6}$ & $\mathbf{7}$ & $\mathbf{A v e r a g e}$ \\
\hline Unspecific phages (U) & 2,880 & 540 & 14,200 & 170 & 1,600 & 1,340 & 400 & \\
\hline Phages binding target (T) & 8,000 & 9,670 & 19,000 & 900 & 13,000 & 46,000 & 800 & \\
\hline T/U & 2.8 & 17.9 & 1.3 & 5.3 & 8.1 & 34.3 & 2 & 10.2 \\
\hline
\end{tabular}


Tab. 3: Reactivity of selected antibodies to antigenic targets

\begin{tabular}{|l|c|c|c|c|c|c|c|c|c|}
\hline Targets & $\mathbf{1}$ & $\mathbf{2}$ & $\mathbf{3}$ & $\mathbf{4}$ & $\mathbf{5}$ & $\mathbf{6}$ & $\mathbf{7}$ & Total & \% of total \\
\hline Nb clones tested & 5 & 3 & 1 & 4 & 3 & 5 & 3 & 24 & 100 \\
\hline Positive & 4 & 2 & 0 & 3 & 3 & 3 & 2 & 17 & 71 \\
\hline Negative & 1 & 1 & 1 & 0 & 0 & 2 & 0 & 5 & 29 \\
\hline Anti-GST & 0 & 0 & 0 & 0 & 0 & 0 & 0 & 0 & 0 \\
\hline
\end{tabular}

nated. A conceptually similar strategy has been reported previously, where phages were selected against biotinylated proteins adsorbed alternatively to neutravidin and to streptavidin beads, to avoid selection of phages binding to neutravidin or streptavidin beads (Stefan et al., 2011). One interesting possibility, not tested here, would be to use successively three types of beads (glutathione, streptavidin, neutravidin) to reduce even further the selection of non-specific phages binding to beads. We further verified the specificity of the selected $\mathrm{scFv}$ fragments by subcloning them in a mammalian expression plasmid and testing the specificity of the antibodies by Western blot and ELISA. Our results confirm that a majority $(17 / 24)$ of selected $\mathrm{scFv}$ fragments presented a high specificity for their target antigen. The remaining fraction (7/24) of antibodies exhibiting no specific binding to the target antigen may correspond to either non-specific antibodies, antibodies that lost their specificity upon expression as Fc fusion proteins in mammalian cells, or antibodies not produced in sufficient quantities in mammalian cells. A detailed analysis of these possibilities may allow further optimization of the selection procedure.

Overall the procedure described in this study allows the simple production of antigenic sequences in bacteria, and selection of antibodies specific for virtually any protein target sequence. Simple methods for in vitro selection of monoclonal antibodies should facilitate the replacement of animal immunization with recombinant antibody technology.

\section{References}

Ahmad, Z. A., Yeap, S. K., Ali, A. M., et al. (2012). scFv antibody: principles and clinical application. Clin Dev Immunol 2012, 980250.

Beckett, D., Kovaleva, E., and Schatz, P. J. (1999). A minimal peptide substrate in biotin holoenzyme synthetase-catalyzed biotinylation. Protein Sci 8, 921-929.

Benghezal, M., Gotthardt, D., Cornillon, S., and Cosson, P. (2001). Localization of the Rh50-like protein to the contractile vacuole in Dictyostelium. Immunogenetics 52, 284-288.

Cornillon, S., Pech, E., Benghezal, M., et al. (2000). Phg1p is a nine-transmembrane protein superfamily member involved in dictyostelium adhesion and phagocytosis. J Biol Chem 275, 34287-34292.

de Haard, H. J., van Neer, N., Reurs, A., et al. (1999). A large nonimmunized human Fab fragment phage library that permits rapid isolation and kinetic analysis of high affinity antibodies. J Biol Chem 274, 18218-18230. de Wildt, R. M., Mundy, C. R., Gorick, B. D., and Tomlinson, I. M. (2000). Antibody arrays for high-throughput screening of antibody-antigen interactions. Nat Biotechnol 18, 989-994.

Graham, F. L. and van der Eb, A. J. (1973). Transformation of rat cells by DNA of human adenovirus 5. Virology 54, 536-539.

Haque, A. and Tonks, N. K. (2012). The use of phage display to generate conformation-sensor recombinant antibodies. Nat Protoc 7, 2127-2143.

Kohler, G. and Milstein, C. (1975). Continuous cultures of fused cells secreting antibody of predefined specificity. Nature 256, 495-497.

Moutel, S., El Marjou, A., Vielemeyer, O., et al. (2009). A multi$\mathrm{Fc}$-species system for recombinant antibody production. BMC Biotechnol 9, 14.

Nissim, A., Hoogenboom, H. R., Tomlinson, I. M., et al. (1994). Antibody fragments from a 'single pot' phage display library as immunochemical reagents. EMBO J 13, 692-698.

Nizak, C., Monier, S., del Nery, E., et al. (2003). Recombinant antibodies to the small GTPase Rab6 as conformation sensors. Science 300, 984-987.

Smith, G. P. (1985). Filamentous fusion phage: novel expression vectors that display cloned antigens on the virion surface. Science 228, 1315-1317.

Stefan, N., Martin-Killias, P., Wyss-Stoeckle, S., et al. (2011). DARPins recognizing the tumor-associated antigen EpCAM selected by phage and ribosome display and engineered for multivalency. J Mol Biol 413, 826-843.

Vodnik, M., Zager, U., Strukelj, B., and Lunder, M. (2011). Phage display: selecting straws instead of a needle from a haystack. Molecules 16, 790-817.

\section{Acknowledgements}

This project was supported by the Doerenkamp-Zbinden Foundation and by the Fondation Egon Naef pour la Recherche In Vitro (FENRIV). The laboratory is supported by a grant from the Swiss National Science Foundation to P. C. (31003A-135789).

\section{Correspondence to}

Pierre Cosson, $\mathrm{PhD}$

Department of Cell Physiology and Metabolism

Centre Médical Universitaire

1 rue Michel Servet

1211, Geneva 4

Switzerland

e-mail: Pierre.Cosson@unige.ch 